\title{
Spin ordering in magnetic quantum dots: From core-halo to Wigner molecules
}

\author{
R. Oszwałdowski, ${ }^{1}$ P. Stano, ${ }^{2}$ A. G. Petukhov ${ }^{3}$ and Igor Žutić ${ }^{1}$ \\ ${ }^{1}$ Department of Physics, University at Buffalo, Buffalo, NY 14260-1500 \\ ${ }^{2}$ Institute of Physics, Slovak Academy of Sciences, 84511 Bratislava, Slovakia and \\ Department of Physics, University of Basel, Klingelberstrasse 82, 4056 Basel, Switzerland \\ ${ }^{3}$ Department of Physics, South Dakota School of Mines and Technology, Rapid City, SD 57701
}

\begin{abstract}
The interplay of confinement and Coulomb interactions in quantum dots can lead to strongly correlated phases differing qualitatively from the Fermi liquid behavior. We explore how the presence of magnetic impurities in quantum dots can provide additional opportunities to study correlation effects and the resulting ordering in carrier and impurity spin. By employing exact diagonalization we reveal that seemingly simple two-carrier quantum dots lead to a rich phase diagram. We propose experiments to verify our predictions, in particular we discuss interband optical transitions as a function of temperature and magnetic field.
\end{abstract}

PACS numbers: 75.50.Pp,75.30.Hx,75.10.Lp,75.10.-b

With high tunability of their parameters, quantum dots (QDs) are ideal systems for exploring correlation effects $\stackrel{\underline{1-4}}{-\underline{4}}$ While in 3 dimensions the correlation-induced Wigner crystal ${ }^{5}$ is elusive and only expected in the limit of an extremely low carrier-density ${ }_{2}^{2.6}$ its nanoscale ana$\log$, the Wigner molecule (WM) $\stackrel{2,3.7}{\longleftarrow}$ was observed in QDs at much higher densities $\frac{8,9}{9}$ An increase in the relative strength of Coulomb interactions qualitatively changes the liquid-like independent-particle picture to that of WM characterized by electron localization and strong angular order $\underline{\underline{3.7 .8}}$

We expect that doping QDs with magnetic impurities ${ }^{10}-13$ (typically Mn) opens unexplored opportunities to study the nanoscale correlations. Through Mn-carrier exchange interaction, the correlations can be enhanced, imprinted on Mn-spins, and thus observed. Several key advances in elucidating correlations in non-magnetic QDs were accomplished in two-electron systems $2,8,9,14$ However, even in simple circular QDs, identifying WMs is complicated by insufficient accuracy in the treatment of correlations 2.14 and artifacts of the mean-field and Hartree-Fock approaches $\stackrel{15}{\underline{15}}$ To understand these systems, the exact diagonalization ${ }^{8.9 .14}$ is particularly suitable, corroborating analytical findings for two-electron correlations in QDs: $\underline{16}, \underline{17}$

Here, we generalize the exact approach ${ }^{18,19}$ to probe the charge and spin densities of carriers as well as Mn-spin ordering in magnetic QDs with two carriers (holes) $\stackrel{20}{\underline{2}}$ To elucidate the stability of the magnetic ordering, we consider different Mn-doping configurations, deformation of circular QD confinement, and examine the effects of temperature $T$ and magnetic field $B$.

The phase diagram in Fig. 1, for Mn-doped circular QDs, shows three magnetic groundstates, to be contrasted with the spin-singlet groundstate of non-magnetic QDs $\stackrel{2}{2}$ The groundstate phase changes with the doping radius $R_{\mathrm{eff}}$ and the fraction, $x_{\mathrm{Mn}}$, of cations replaced by Mn-atoms in the QD. The carrier spin density is imprinted on Mn-spins forming three patterns corresponding to: two pseudo-singlets (PS) characterized by total hole-spin zero, but non-zero hole-spin density $\stackrel{21}{\longleftarrow}$ and a spin triplet (T), see Fig. 1(a-c).

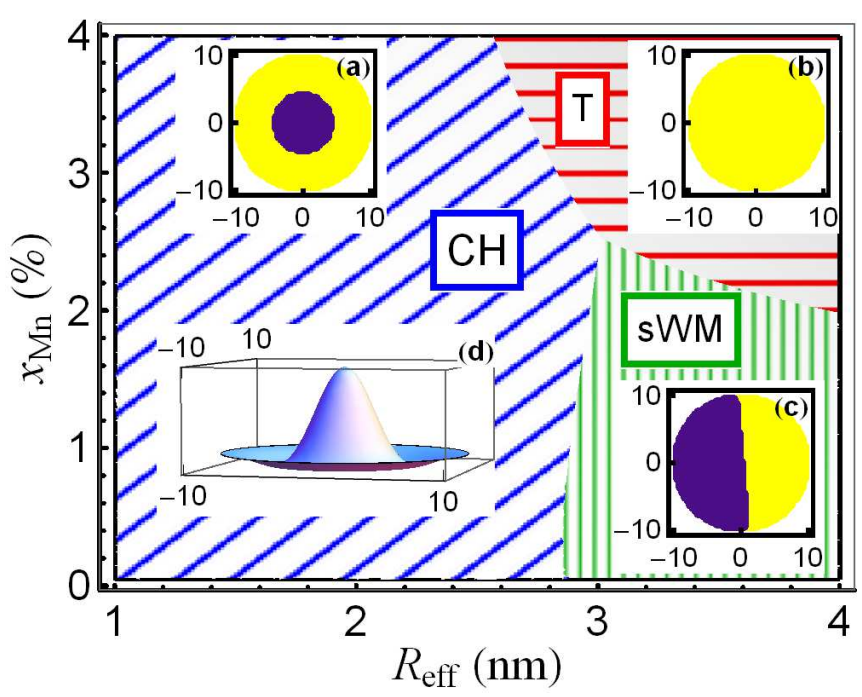

FIG. 1. (color online) Groundstate phase diagram (PS vertical and skew, T horizontal hatching) as a function of Mncontent $x_{\mathrm{Mn}}$ and doping radius $R_{\text {eff }}$, for a double-occupied circular dot, $\hbar \omega_{x, y}=25 \mathrm{meV}$, at zero temperature. Insets (a-c): QD-top view (in-plane coordinates in $\mathrm{nm}$ ) of Mn-spin patterns; spins up: light, down: dark. (a) Core-halo $(\mathrm{CH})$ : $R_{\text {eff }}=2 \mathrm{~nm}, x_{\mathrm{Mn}}=2 \%$, (b) triplet $(\mathrm{T}): R_{\text {eff }}=3.5 \mathrm{~nm}$, $x_{\mathrm{Mn}}=3 \%$, (c) spin-Wigner Molecule (sWM): $R_{\mathrm{eff}}=3.5 \mathrm{~nm}$, $x_{\mathrm{Mn}}=1 \%$, (d) Hole-spin density for the pattern in (a) ${ }^{22}$

Before we provide a detailed analysis, it is instructive to view the PS state in Fig. 1(c) as the spin-WM. In nonmagnetic circular QDs, their WM "dimer-like" phase can be fully revealed only in the pair-correlation function $\stackrel{17}{ }$ However, a similar phase can be directly detected in magnetic QDs: the Mn-pattern of spin-WM reflects a double-peaked hole-spin density. The separation between

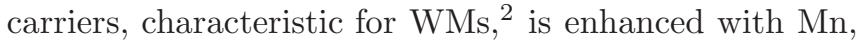
which provides a spin structure.

We use the total QD Hamiltonian, $\hat{H}=\hat{H}_{f}+\hat{H}_{\text {ex }}$, with 
typical 2D non-magnetic and exchange parts, $\stackrel{21}{=}$ where

$$
\hat{H}_{f}=\sum_{i=1,2}\left[\frac{\boldsymbol{\pi}_{i}^{2}}{2 m^{*}}+\frac{m^{*}}{2}\left(\omega_{x}^{2} x_{i}^{2}+\omega_{y}^{2} y_{i}^{2}\right)\right]+\frac{e^{2} / 4 \pi \epsilon}{\left|\boldsymbol{r}_{1}-\boldsymbol{r}_{2}\right|},
$$

the holes are labeled by $i, m^{*}$ is the effective mass, $e$ the electron charge, and $\epsilon$ the dielectric constant, momentum $\pi_{i}$ includes the vector potential of field $B \| z z^{23}$ The $p-$ $d$ exchange interaction between $\mathrm{Mn}$-spins and confined holes has the Ising form 24

$$
\hat{H}_{\mathrm{ex}}=-(\beta / 3) \sum_{i=1,2} \sum_{j=1}^{N} \hat{s}_{i z} \hat{S}_{j z} \delta\left(\mathbf{r}_{i}-\mathbf{R}_{j}\right),
$$

because of the strong $z$-axis anisotropy, arising from spinorbit interaction in the $2 \mathrm{D}$ QDs. Here, $\beta$ is the exchange constant, $\hat{s}_{z}$ is the heavy-hole pseudospin with projections $s_{z}= \pm 3 / 2$, while $S_{z}$ are operators of $z$-projection of Mn-spins positioned at $\mathbf{R}_{j}$, and $N$ is the number of Mn-spins in the dot.

Since $\hat{H}_{\text {ex }}$ does not contain spin-flip processes, the total wavefunction is a product of the hole and Mnspin parts. The partition function of the system can be calculated using Gibbs canonical distribution, $Z=$ $\operatorname{Tr}_{S_{j z}} \sum_{n} \exp \left[-E_{n}\left(\left\{S_{j z}\right\}\right) / k_{B} T\right]$, where $k_{B}$ is the Boltzmann constant, and the hole eigenvalues, $E_{n}$, depend on $N$ numbers $S_{j z}$, with each $S_{j z}=-S, \ldots, S=5 / 2$ (index $n$ runs over hole states for fixed $\left\{S_{j z}\right\}$ ). To calculate $Z$, one would need to solve $6^{N}$ replicas of the hole Schrödinger equation, with $N \sim 10^{2}-10^{3}$.

We can overcome this obstacle of computational complexity by partitioning the dot into $N_{c}$ square cells, each containing few $\mathrm{Mn}$-spins, and neglecting spatial variation of the two-hole wavefunction $\Phi_{n}$ within each cell through the use of the average hole-spin density

$$
\left\langle s_{k}\right\rangle_{n}=\frac{1}{h_{z} N_{k}} \sum_{j \in N_{k}}\left\langle\Phi_{n}\left|\sum_{i} \hat{s}_{i z} \delta\left(\mathbf{r}_{i}-\mathbf{R}_{j}\right)\right| \Phi_{n}\right\rangle,
$$

where $h_{z}$ is the QD height. For a given cell with $N_{k}$ spins $S_{j z}^{(k)}$ creating a magnetic moment $M_{k}$, a distribution function of the average dimensionless magnetization, $m_{k} \equiv-M_{k} / g_{\mathrm{Mn}} \mu_{B} N_{k}$, can be expressed as $Y\left(m_{k}\right) \propto$ $\exp \left[-G_{k}\left(m_{k} / S\right) / k_{B} T\right]$. Here $g_{\mathrm{Mn}}=2$ is the Mn $g$-factor. The Gibbs free energy of the $N_{k}$ non-interacting spins, $G_{k}\left(m_{k} / S\right)$, is obtained by Legendre transformation, 25

$$
\frac{G_{k}(x)}{N_{k} k_{B} T}=\left[x B_{S}^{-1}(x)-\ln \frac{\sinh \left[(1+1 / 2 S) B_{S}^{-1}(x)\right]}{\sinh \left[(1 / 2 S) B_{S}^{-1}(x)\right]}\right],
$$

where $B_{S}^{-1}$ is the inverse of the Brillouin function $B_{S}$. Using $Y\left(m_{k}\right)$, we transform $Z$ with exponential accuracy as

$$
Z \propto \sum_{n} \int \ldots \int \exp \left[-G_{\mathrm{tot}}^{n} / k_{B} T\right] \prod_{k=1}^{N_{c}} d m_{k}
$$

where $G_{\text {tot }}^{n}=\sum_{k} G_{k}\left(m_{k} / S\right)+E_{n}\left(\left\{m_{k}\right\}\right)$. For any $n$, each integral in Eq. (5) can be evaluated using the steepest descent method. Equation for the saddle point, combined with the HellmannFeynman theorem, $-\beta N_{k}\left\langle s_{k}\right\rangle / 3+g_{\mathrm{Mn}} N_{k} \mu_{B} B=$ $\partial E_{n}\left(m_{1}, \ldots, m_{k}, \ldots, m_{N_{c}}\right) / \partial m_{k}$, leads to the selfconsistency condition

$$
m_{k}=S B_{S}\left[S\left(\beta\left\langle s_{k}\right\rangle_{n} / 3-g_{\mathrm{Mn}} \mu_{B} B\right) / k_{B} T\right] .
$$

Our analysis shows that the self-consistency condition Eq. (6) depends on the quantum-mechanical average, $\left\langle s_{k}\right\rangle_{n}$, relevant for small systems such as QDs, rather than on the thermal average, $\stackrel{25}{\stackrel{2}{r}}$ thereby avoiding artifacts arising from imposing the thermodynamic limit on a nanoscale system. We use Eq. (6) to find a global minimum of $G_{\text {tot }}^{0}\left(\left\{m_{k}\right\}\right)$ corresponding to the groundstate, where 0 stands for PS or $\mathrm{T}$ in different regions of the phase diagram.

We find the eigenstates of $\hat{H}$ self-consistently: For fixed values of $m_{k}$ (randomly initialized), we obtain intermediate two-hole states. Since $\left[\hat{H}, \hat{\Sigma}_{z}\right]=0$, where $\hat{\Sigma}_{z}$ is the total hole-spin $z$-projection, the states are either in PS $\left(\Sigma_{z}=0\right)$ or $\mathrm{T}\left(\Sigma_{z}= \pm 3\right)$ orthogonal subspaces $\stackrel{21}{\underline{ } \mathrm{Pe}}$ choose the lowest state in each subspace, use Eq. (6) to obtain new $m_{k}$, and restart exact diagonalization, iterating until convergence. The groundstate nature, either $\mathrm{T}$ or PS, depends on the QD parameters (for $x_{\mathrm{Mn}}=0=B$ the groundstate is a singlet).

We use ZnTe parameters: hole mass $m^{*}=0.2$ electron

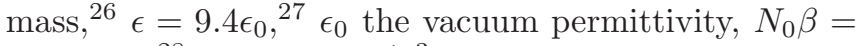
$-1.05 \mathrm{eV}, \frac{28}{r}$ and $N_{0}=4 / a^{3}$ the cation density with the lattice constant $a \simeq 6.1 \AA$. For typical self-assembled QDs: $\hbar \omega_{x, y}=10-30 \mathrm{meV}, x_{\mathrm{Mn}} \leq 5 \%$, our standard values are $x_{\mathrm{Mn}}=1 \%, h_{z}=1.8 \mathrm{~nm}, \hbar \omega_{0} \equiv \hbar \omega_{x, y}=$ $25 \mathrm{meV}$, corresponding to a characteristic length $29 l_{0}=$ $\left(\hbar / m^{*} \omega_{0}\right)^{1 / 2}=3.9 \mathrm{~nm}$.

We now revisit Mn-patterns in Fig. 1 calculated at $T=0$, with a doping profile $x_{\mathrm{Mn}}\{1+$ $\left.\exp \left[\left(r-R_{\text {eff }}\right) / \xi\right]\right\}^{-1}$ of width $\xi=0.25 \mathrm{~nm}$, and radius $R_{\text {eff }}$, here $r$ is the distance from the center. In addition to the triplet groundstate (all Mn-spins parallel) at relatively large $R_{\text {eff }}$ and $x_{\mathrm{Mn}}$, and the spin-WM groundstate at smaller $x_{\mathrm{Mn}}$, another PS forms, Fig. 1(a), we term it "core-halo", $\underline{30}$ Inset (d) shows the resulting average spindensity $\left\langle s_{k}\right\rangle$. In contrast to spin-WM, the Mn-pattern and $\left\langle s_{k}\right\rangle$ for core-halo preserve the circular symmetry of the QD. For $\xi \rightarrow 0$ (no Mn for $r>R_{\text {eff }}$ ) and small enough $R_{\text {eff }}$ (e.g. $R_{\text {eff }}=2 \mathrm{~nm}, x_{\mathrm{Mn}}=1 \%$ ) the core-halo patterns become purely "ferromagnetic." We find that such unidirectional patterns form also for other inhomogeneous $x_{\mathrm{Mn}}$-profiles, confirming predictions from Ref. $\underline{21}$

In the $R_{\text {eff }} \rightarrow \infty$ regime, we study stability of $\mathrm{T}$ and PS, termed magnetic bipolarons $\stackrel{21}{2}$ Figure 2 (a) shows that, already at modest $x_{\mathrm{Mn}}$, the Mn-induced energy gain of PS exceeds that of a magnetic polaron forming for a single carrier 31 This suggests that the robustness of magnetic bipolarons is similar to that of the well-established single magnetic polarons. 


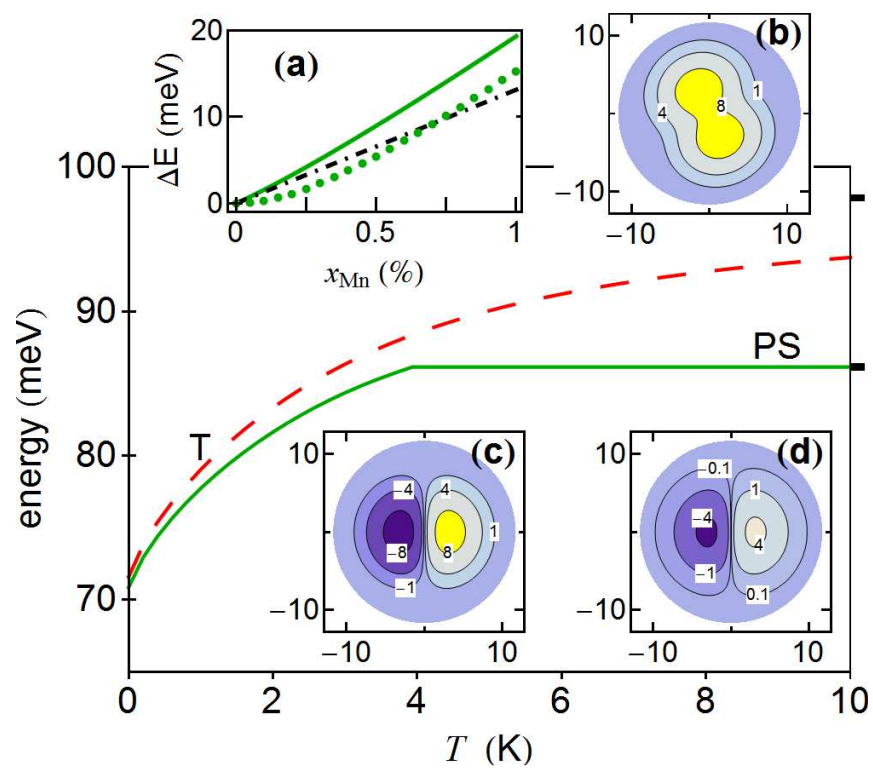

FIG. 2. Temperature evolution of pseudosinglet $E_{\mathrm{PS}}$ (green) and triplet $E_{\mathrm{T}}$ (red) energies for a circular QD, $\hbar \omega_{x, y}=25$ $\mathrm{meV}$, and $x_{\mathrm{Mn}}=1 \%$. Bold tics show the high- $T$ (zero $p-d$ exchange) limit. (a): Mn-induced energy gains at $T=0$ K. Dots/solid line: numerical/variational results ${ }^{32}$ for PS, $\Delta E_{\mathrm{PS}}=E_{\mathrm{PS}}\left(x_{\mathrm{Mn}}=0\right)-E_{\mathrm{PS}}$. Dash-dotted line: single magnetic polaron $\Delta E_{\mathrm{MP}}=x_{\mathrm{Mn}} N_{0}|\beta| S / 2 . \Delta E_{\mathrm{MP}}<\Delta E_{\mathrm{PS}}$ for $x_{\mathrm{Mn}} \geq 0.7 \%$. Insets (b-d): $m_{k}$ in units of $1 / 4$ as a function of position (nm); (b) triplet at $1 \mathrm{~K},(\mathrm{c}, \mathrm{d})$ PS at $1 \mathrm{~K}, 3 \mathrm{~K}$.

Figure 2 shows the temperature dependence of bipolaron energies. Owing to the stronger exchange interaction, the triplet approaches the high- $T$ limit at a higher $T$ than PS. The asymptotic trend $-1 / T$ in $E_{\mathrm{T}}$, typical for paramagnets, is expected since the effective exchange field of triplet holes, acting on $\mathrm{Mn}$, is nearly independent of the Mn-spin alignment. In contrast, PS has a secondorder transition to singlet ${ }^{32}$ Insets (b-d) show the magnetization [Eq. (6)] of PS and T . Finite temperature has a different effect on the two states. For PS, $m_{k}$ retains its overall $0 \mathrm{~K}$ shape, and decreases uniformly. In contrast, the saturated Mn-pattern of T, Fig. T(b), undergoes a transition to a state with spontaneously broken circular symmetry: two symmetrical and unidirectional peaks appear, Fig. 2(b). The peaks reflect the hole-spin density, which maximizes the Mn-induced energy gain through a linear combination of the two triplets with opposite angular momenta. $\frac{12}{2}$

Having established the presence of magnetic bipolarons and Mn-patterns in circular QDs, it is crucial to examine the more realistic case of asymmetric confinement. We introduce in-plane asymmetry through $\omega_{x, y}=$ $\hbar /\left(m^{*} l_{x, y}^{2}\right)$, where $l_{x, y}=l_{0}(1+d)^{ \pm 1} \underline{\underline{34}}$ The asymmetry stabilizes the spin-WM along the weaker-potential axis, Fig. 3(a), since separating the holes in the direction of the "softer" potential costs less potential energy. As in circular QDs [Fig. [1, large $R_{\text {eff }}$ ], the triplet becomes the groundstate with increasing $x_{\mathrm{Mn}}$. For increasing asym-

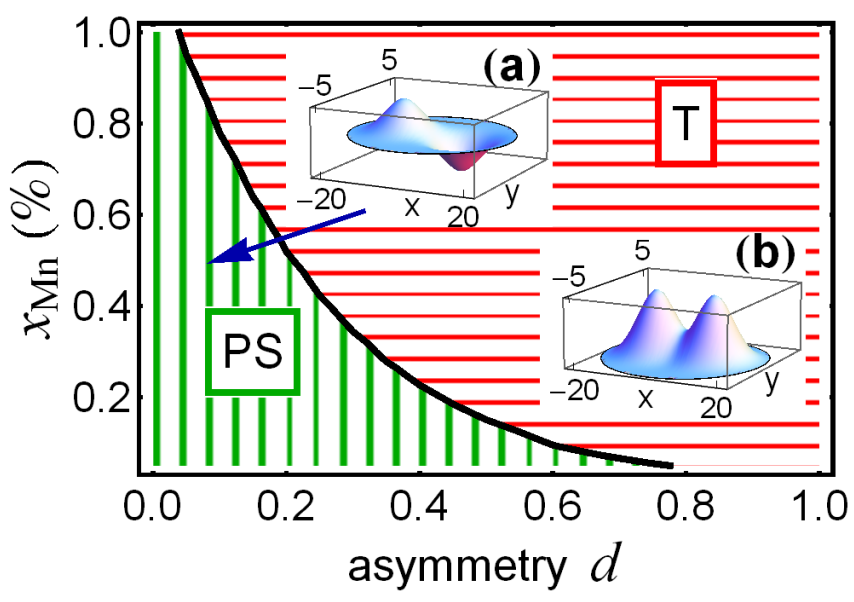

FIG. 3. (color online) Groundstate phase diagram (PS vertical, T horizontal hatching) as a function of confinement asymmetry $d$ and $x_{\mathrm{Mn}}$, at $T=0$. Insets: hole spin density of PS (a) and $\mathrm{T}(\mathrm{b}), \hbar \omega_{0}=25 \mathrm{meV}, d=1$, and $x_{\mathrm{Mn}}=1 \%$.

metry $d$, the non-magnetic singlet-triplet gap decreases, so that $\mathrm{T}$ becomes the groundstate at lower $x_{\mathrm{Mn}} \underline{\underline{33}}$

The results so far can be understood introducing an effective, spin-dependent interaction between the two holes. Each hole tends to polarize Mn-spins within an area defined by temperature and the confinement. Consequences of the overlap of the areas depend on relative hole spins. If they are antiparallel (PS), mutual repulsion arises, $\stackrel{32}{=}$ which would lead to complete separation of holes in absence of the confinement (magnetic bipolaron would not form). In contrast, holes with parallel spins $(\mathrm{T})$ effectively attract each other,$\frac{35}{5}$ as they benefit from sharing the cloud of polarized Mn-spins each of them carries $\stackrel{32}{\underline{3}}$

Opposite limits of the doping radius $R_{\text {eff }}$ offer a simple insight as to which of the two PS patterns is the groundstate. For $R_{\text {eff }} \rightarrow 0$, the $p-d$ exchange energy gain for spin-WM is zero, since this state produces $\left\langle s_{k}\right\rangle=0$ at the QD center. On the other hand, for $R_{\text {eff }} \rightarrow \infty$ and for weak confinement, the proper limit is that of two separate, localized magnetic polarons, a scenario consistent with spin-WM rather than core-halo $\underline{\underline{32}}$

To corroborate our predictions, as well as other works considering magnetic interactions in closed-shell QDs, $\stackrel{13,21,36,37}{2}$ we propose experiments that test the existence of magnetic bipolarons, and discriminate the different Mn-patterns. One such probe is interband photoluminescence, $10,11,38$ With a sufficiently intense excitation, two kinds of emission lines appear, corresponding to $2 \rightarrow 1$ and $1 \rightarrow 0$ QD-occupancy transitions. We calculate the photon energies, $E_{\mathrm{ph}}$, for the standard parameters, assuming: (i) type-II band alignment, $\underline{28,32}$ (ii) Mn-spin pattern does not change during a recombination event, (iii) the system recombines from its (two- or single-hole) groundstate. We show that $E_{\mathrm{ph}}$ dependence on $T$ and $B$ allows to identify the bipolarons.

We first consider varying $T$, Fig. (44). Single polaron, 
characterized by a unidirectional Mn-pattern, shows a $1 / T$ redshift. In contrast, thermal disruption of PS occurs with an abrupt change of the slope of $E_{\mathrm{ph}}$ at few kelvin.

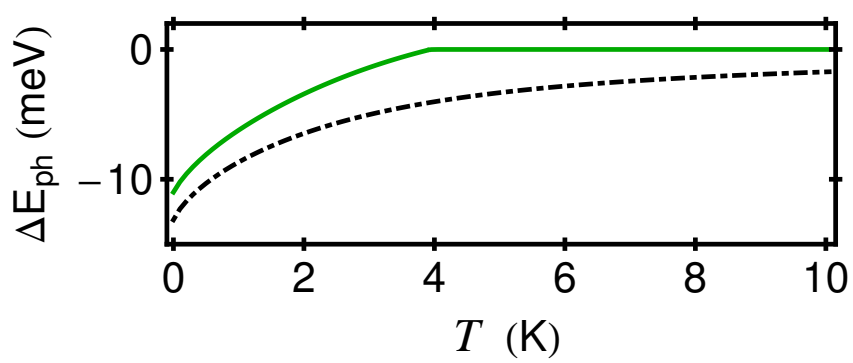

FIG. 4. (color online) Temperature dependence of the PS $\rightarrow 1$ hole (solid), and $1 \rightarrow 0$ (dash-dotted) transition energies $E_{\mathrm{ph}}$, for $B=0$. To better compare the dependencies, each line is shifted: $\Delta E_{\mathrm{ph}} \equiv E_{\mathrm{ph}}(T)-E_{\mathrm{ph}}(T \rightarrow \infty)$.

We next consider $B \| z$ (Faraday configuration 28,39 ). For a triplet, $m_{k} \|-B$ everywhere. The small change of $\Delta E_{\mathrm{ph}}$, (Fig. 5] dashed) is mainly due to orbital effects, since $m_{k}$, highly saturated at the low- $T$, is not very sensitive to $B$. For PS, a part of Mn-spins is aligned unfavorably, i.e., parallel to $B$. Increasing $B$ changes their projection [Fig. $[5(\mathrm{a}, \mathrm{b})]$. The accompanying change of $E_{\mathrm{PS}}$ (not shown) becomes abrupt at a threshold $B_{0}$ (the Mn-pattern becomes unidirectional at $B_{0}$ ), and then flattens out close to its asymptotic value: the non-magnetic singlet energy $\stackrel{40}{=}$ For $B>B_{0}\left(B_{0} \simeq 1.5 \mathrm{~T}\right.$ in Fig. 5), the $B$-dependence of PS $\rightarrow 1$ transition energies is dominated by changes of final-state energies, which react to increasing saturation of $m_{k}$. The line is split by the field, as the remaining hole can end up in two opposite spin states. No such splitting occurs for the $\mathrm{T} \rightarrow 1$ and $1 \rightarrow 0$ transitions. Thus, the splitting, and the abrupt 'melting' at $B_{0}$, signal the PS groundstate.

Finally, the two possible PS states can be resolved using selection rules for photoluminescence. $\underline{41}$ The circularly symmetric core-halo pattern forbids recombination with $p$-like excited electron states. However, such transitions are (weakly) allowed for spin-WM, 32 and would appear at a strong optical pumping. ${ }^{42}$ As an alternative to photoluminescence, scanning tips with NV-centers could offer sufficient spatial sensitivity to probe the Mn-spin patterns $\underline{43}$
We expect that our findings will motivate future efforts to probe magnetization patterns and correlation effects at the nanoscale. Previously unexplored regimes are afforded with Mn-doping. Effective internal magnetic fields in colloidal QDs can reach $\sim 100 \mathrm{~T}, 11$ beyond what is feasible with applied static magnetic fields. Consistent with recent advances in the field of nanomagnetism, 44 an increasing number of experimental probes are likely to meet the challenge of detecting the predicted spin ordering even in single QDs.

Nuclear spins in few-electron III-V QDs could provide a magnetically-active feedback similar to that stud-

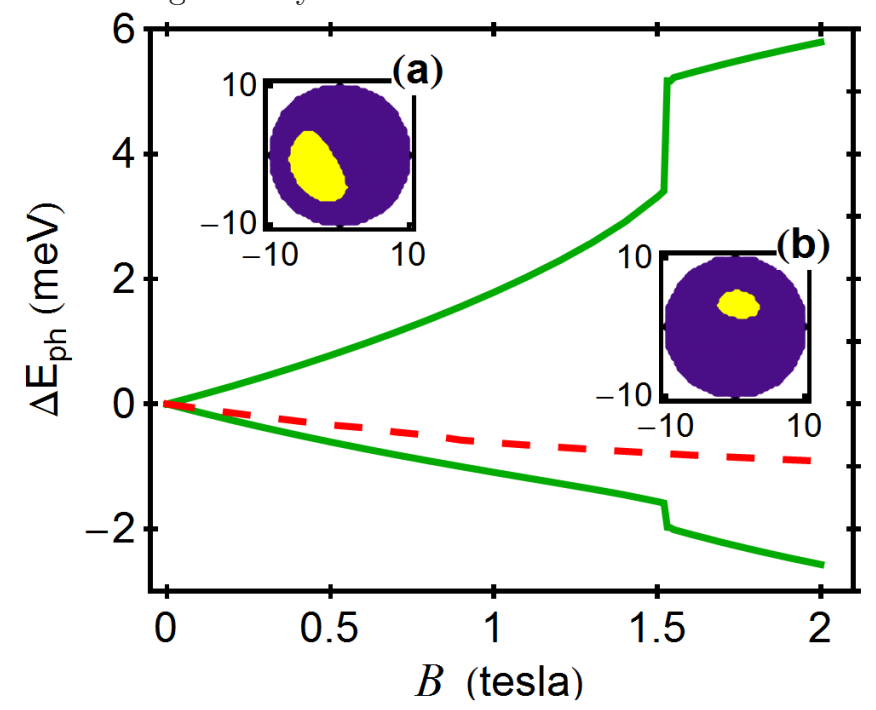

FIG. 5. (color online) $B \| z$ dependence of the PS $\rightarrow 1$ (solid lines) and $\mathrm{T} \rightarrow 1$ (dashed), for $T=1 \mathrm{~K}$. To avoid PS-T crossing, we set $x_{\mathrm{Mn}}=0.5 \%$. Each line is shifted: $\Delta E_{\mathrm{ph}} \equiv$ $E_{\mathrm{ph}}(B)-E_{\mathrm{ph}}(B=0)$. Insets: PS Mn-patterns for 0.4 (a), and 1.4 tesla (b); light (dark) for $m_{k}>0\left(m_{k}<0\right)$.

ied here. While the electron-nuclear spin interaction is weak, leading to a much smaller temperature scale for analogous polaron objects, this scale is known to be very strongly enhanced by electron-electron interactions in low-dimensional systems $\underline{45}$

This work was supported by DOE-BES, ONR, metaQUTE ITMS NFP 26240120022, CE SAS QUTE, EU Project Q-essence, APVV-0646-10 and SCIEX.
1 S. M. Reimann and M. Manninen, Rev. Mod. Phys. 74, 1283 (2002).

2 C. Yannouleas and U. Landman, Rep. Prog. Phys. 70, 2067 (2007).

3 A. Ghosal, A. D. Guclu, C. J. Umrigar, D. Ullmo, and H. U. Baranger, Nature Phys. 2, 336 (2006).

4 R. Hanson et al., Rev. Mod. Phys. 79, 1217 (2007); L. P. Kouwenhoven, D G. Austig, and S. Tarucha, Rep. Prog.
Phys. 64, 701 (2001); S. Maekawa (Ed.) Concepts in Spin Electronics (Oxford University Press, Oxford, 2006).

${ }^{5}$ E. Wigner, Phys. Rev. 46, 1002 (1934).

${ }^{6}$ P. Fulde, Electron Correlations in Molecules and Solids (Springer, Berlin, 1993).

7 R. Egger, W. Häusler, C. H. Mak, and H. Grabert, Phys. Rev. Lett. 82, 3320 (1999).

8 A. Singha et al., Phys. Rev. Lett. 104, 246802 (2010). 
9 C. Ellenberger et al., Phys. Rev. Lett. 96, 126806 (2006).

10 J. Seufert et al., Phys. Rev. Lett. 88, 027402 (2001); L. Besombes, et al., ibid. 93, 207403 (2004); F. Xiu et al. ACS Nano 4, 4948 (2010); S. T. Ochsenbein et al., Nat. Nanotech. 4, 68 (2009); R. Viswanatha et al., Phys. Rev. Lett. 107, 067402 (2011); F. Henneberger and J. Puls, in Introduction to the Physics of Diluted Magnetic Semiconductors edited by J. Kossut and J. A. Gaj (Springer, Berlin, 2010); I. A. Merkulov and A. V. Rodina, ibid.; Ł. Kłopotowski et al., Phys. Rev. B 83, 081306(R) (2011); A. A. Maksimov et al., ibid. 62, R7767 (2000); N. T. T. Nguyen and F. M. Peeters, ibid. 78, 045321 (2008); J. M. Pientka, R. Oszwałdowski, A. G. Petukhov, J. E. Han, and I. Žutić, ibid. 86, 161403 (R) (2012); R. M. Abolfath, P. Hawrylak, and I. Žutić, Phys. Rev. Lett. 98, 207203 (2007).

11 R. Beaulac, L. Schneider, P. I. Archer, G. Bacher, and D. R. Gamelin, Science 325, 973 (2009).

12 A. O. Govorov, Phys. Rev. B. 72, 075359 (2005); C.R. Physique 9, 857 (2008).

13 J. Fernández-Rossier and L. Brey, Phys. Rev. Lett. 93, 117201 (2004); F. Qu and P. Hawrylak, ibid. 96, 157201(2006).

14 D. Pfannkuche, V. Gudmundsson, and P. A. Maksym, Phys. Rev. B 47, 2244 (1993).

15 For example, the spontaneous symmetry breaking, incorrect spin configurations, and spurious radial dependence of the carrier wavefunction,,-7 L. Serra, R. G. Nazmitdinov, and A. Puente, Phys. Rev. B 68, 035341 (2003).

16 U. Merkt, J. Huser, and M. Wagner, Phys. Rev. B 43, 7320 (1991).

17 M. Taut, Phys. Rev. A 48, 3561 (1993).

18 By systematically including the effects of carrier-Mn interactions, we generalize the method shown to be very accurate for non-magnetic QDs, F. Baruffa, P. Stano, and J. Fabian, Phys. Rev. Lett. 104, 126401 (2010); P. Stano and J. Fabian, ibid. 96, 186602 (2006).

19 F. Baruffa, P. Stano, and J. Fabian, Phys. Rev. B 82, 045311 (2010).

20 The choice of holes, rather than electrons, leads to enhanced carrier-Mn exchange interaction. Interesting results for magnetic bipolarons based on electrons are presented in H. Bednarski and J. Spałek, J. Phys.: Condens. Matter. 24, 235801 (2012).

21 R. Oszwałdowski, I. Žutić, and A. G. Petukhov, Phys. Rev. Lett. 106, 177201 (2011).

${ }^{22}$ R. Oszwałdowski, invited APS presentation, http://meetings.aps.org/link/BAPS 2012.MAR.P14.1.

23 The action of $B$ on magnetic moments of holes is negligible for the effects described here.

${ }^{24}$ K. Vyborny, J. E. Han, R. Oszwałdowski, I. Žutić and A. G. Petukhov, Phys. Rev. B 85, 155312 (2012); P. S. Dorozhkin et al., ibid. 68, 195313 (2003).

25 A. G. Petukhov, I. Žutić, and S. C. Erwin, Phys. Rev. Lett. 99, 257202 (2007).

${ }^{26}$ Y. Oka and M. Cardona, Phys. Rev. B 23, 4129 (1981).

27 H. Wagner et al., J. Cryst. Growth 117, 303 (1992).

${ }^{28}$ I. R. Sellers et al., Phys. Rev. B 82, 195320 (2010).

29 The importance of $x_{\mathrm{Mn}}=0$ correlations can be estimated from the Wigner parameter $R_{W}=\left(e^{2} / \epsilon l_{0}\right) / \hbar \omega_{0} \stackrel{2}{2}$

30 The actual orientation of spins in core-halo and spin-WM patterns, as well as the azimuthal angle of the division line in the latter, are not determined by $\hat{H}$, but pseudorandomly in the numerical procedure.

${ }^{31} \mathrm{D}$. R. Yakovlev and W. Ossau, in Introduction to the
Physics of Diluted Magnetic Semiconductors edited by J. Kossut and J. A. Gaj (Springer, Berlin, 2010); T. Dietl and J. Spałek, Phys. Rev. B 28, 1548 (1983).

32 See the auxiliary material.

33 The phase boundaries (Figs. 1 and 3) result from interplay of the single-particle confinement, Coulomb interaction, and magnetic exchange. The phase boundaries will shift in function of the QD parameters.

34 Equivalent to the convention from ${ }^{1}$ and R. M. Abolfath, A. G. Petukhov, and I. Žutić, Phys. Rev. Lett. 101, 207202 (2008).

35 S. Goupalov and A. Kavokin, Solid State Commun. 97, 77 (1996).

36 M. V. Milovanović, E. Dobardžić, and Z. Radović, Phys. Rev. B 80, 125305 (2009); A. Petković, M. V. Milovanović, Phys. Rev. Lett. 98, 066808 (2007).

37 Within the local density approximation, Mnmagnetization patterns appear also for higher QD occupancies (circular QDs and homogeneous Mn doping), R. Abolfath, M. Korkusinski, T. Brabec, and P. Hawrylak, Phys. Rev. Lett. 108, 247203 (2012).

38 K. Katayama, K. Miyajima, M. Ashida, and T. Itoh J. Phys.: Condens. Matter 24, 325801 (2012).

39 I. Žutić, J. Fabian, and S. Das Sarma, Rev. Mod. Phys. 76, 323 (2004).

40 For $T=0$ (full saturation), $E_{\mathrm{PS}}$, as well as the final-state (single-hole) energies are constant above $B_{0}$.

41 M. A. Cusack, P. R. Briddon, and M. Jaros, Phys. Rev. B 56, 4047 (1997); S. Raymond, X. Guo, J. L. Merz, and S. Fafard, ibid. 59, 7624 (1999).

${ }^{42}$ To see if the forbidden transitions are not due to low confinement symmetry, E. Siebert et al., Phys. Rev. B 79, 205321 (2009), the experiment may be repeated at a temperature, at which magnetic bipolarons no longer exist.

${ }^{43}$ L. W. Molenkamp, private communication; J. R. Maze et al., Nature 455, 644 (2008).

44 S. D. Bader, Rev. Mod. Phys. 78, 1 (2006).

45 B. Braunecker, P. Simon, and D. Loss, Phys. Rev. B 80, 165119 (2009); P. Simon, B. Braunecker, and D. Loss, ibid. 77, 045108 (2008). 


\section{Spin Ordering in Magnetic Quantum Dots: From Core-halo to Wigner Molecules (Auxiliary Material)}

This material provides details helping to explain some statements in the main text. The 1st occurrence of the EPAPS reference in the main text points to Sec. A1, the 2nd and 4th to Sec. A1.1, the 3rd to Sec. A1.2, the 5th and 6th to Sec. A2.

\section{A1. VARIATIONAL CALCULATION}

We build the pseudo-singlet (PS) for the spin-WM ground state as

$$
\begin{aligned}
\Phi_{\mathrm{PS}}=\frac{1}{\sqrt{2}} & {\left[\varphi_{u}\left(\boldsymbol{r}_{1}\right) \varphi_{d}\left(\boldsymbol{r}_{2}\right) \chi_{\uparrow}(1) \otimes \chi_{\downarrow}(2)\right.} \\
& \left.-\varphi_{u}\left(\boldsymbol{r}_{2}\right) \varphi_{d}\left(\boldsymbol{r}_{1}\right) \chi_{\uparrow}(2) \otimes \chi_{\downarrow}(1)\right],
\end{aligned}
$$

from normalized orbitals

$$
\begin{aligned}
& \varphi_{u}(\boldsymbol{r})=\frac{1}{L} \sqrt{\frac{2}{\pi}} \exp \left[-\frac{\left(x-X_{0}\right)^{2}+y^{2}}{L^{2}}\right], \\
& \varphi_{d}(\boldsymbol{r})=\frac{1}{L} \sqrt{\frac{2}{\pi}} \exp \left[-\frac{\left(x+X_{0}\right)^{2}+y^{2}}{L^{2}}\right] .
\end{aligned}
$$

Here $\chi_{\sigma}(1)$ and $\chi_{\sigma}(2)$ are spinors of the carriers 1 and 2 , and $\sigma=\uparrow, \downarrow$, while $\boldsymbol{r}=(x, y)$. There are two variational parameters: $L$ and $X_{0}$. The former, width $L$, is introduced to lower the variational energy. The role of the latter, displacement $X_{0}$, is more important: the exchange interaction between holes and Mn spins takes place only for $X_{0} \neq 0$. This parameter introduces separation of the holes with opposite spins, and is important for building the intuitive picture of effective interaction discussed in the main text and below. However, the circular symmetry can be broken in any direction, for example, the $x$-axis can be the division line between Mn spins up and down, this would be reflected by the following replacement

$$
\left(x \pm X_{0}\right)^{2}+y^{2} \rightarrow x^{2}+\left(y \pm Y_{0}\right)^{2}
$$

in Eqs. A2 A3 .

The energy without the $p-d$ exchange is

$$
\begin{array}{r}
E_{\text {nonm }}\left(L, X_{0}\right)=\frac{2 \hbar^{2}}{m^{*} L^{2}}+\frac{1}{2} m^{*} \omega_{0}^{2}\left(L^{2}+2 X_{0}^{2}\right) \\
+\frac{L_{1}}{L} \sqrt{\pi R y^{*} \hbar \omega_{0}} \exp \left(-2 \frac{X_{0}^{2}}{L^{2}}\right) I_{0}\left(2 \frac{X_{0}^{2}}{L^{2}}\right),
\end{array}
$$$$
\text { where } L_{1}=\sqrt{2} l_{0}=\sqrt{\frac{2 \hbar}{m^{*} \omega_{0}}} \text {. }
$$

Here, $m^{*}$ is the effective mass, and $\hbar \omega_{0}$ is the energy quantum of the 2D harmonic oscillator. The second line of Eq. (A4) introduces the Coulomb repulsion of holes, integrated using 2D Fourier transforms [A] $], I_{0}$ is the modified Bessel function of the 1st kind, $R y^{*}=$ $m^{*} e^{4} /\left[32(\pi \epsilon \hbar)^{2}\right]$ is the effective Rydberg, $e$ is the electron charge and $\epsilon$ is the dielectric constant. We calculate the exchange energy using

$$
E_{\mathrm{ex}}=-\Delta_{0} \int\left|\rho_{\mathrm{s}}(\boldsymbol{r})\right| d^{2} r,
$$

(Eq. (A7) in Ref. [A2]). Here, $\Delta_{0}=x_{\mathrm{Mn}} N_{0}|\beta| 5 / 2$ is the exchange splitting, expressed through the fraction of cations replaced by $\mathrm{Mn}$ atoms, $x_{\mathrm{Mn}}$, and the exchange constant $N_{0} \beta<0$. The $2 \mathrm{D}$ spin density, $\rho_{\mathrm{s}}$, is expressed in $\hbar$ units. It is related to $\left\langle s_{k}\right\rangle$ in the main text through $\bar{\rho}_{\mathrm{s}}\left(\boldsymbol{r}_{k}\right) / h_{z}=\left\langle s_{k}\right\rangle / 3$, where bar denotes spatial averaging over a cell $N_{k}$, and $h_{z}$ is the QD height. In the case of PS, the hole spin density is

$$
\rho_{\mathrm{PS}}=\left[\varphi_{u}^{2}(\boldsymbol{r})-\varphi_{d}^{2}(\boldsymbol{r})\right] / 2 .
$$

One finds $\int \rho_{\mathrm{PS}} d^{2} r=0$ owing to normalization of $\varphi_{u, d}(\boldsymbol{r})$. We obtain the exchange energy

$$
E_{\text {ex }}\left(L, X_{0}\right)=-\Delta_{0} \operatorname{erf}\left(\frac{\sqrt{2}\left|\mathrm{X}_{0}\right|}{\mathrm{L}}\right),
$$

where erf is the error function. The results in Fig. (2a main text) are obtained by minimizing

$$
E_{\text {tot }}=E_{\text {nonm }}\left(L, X_{0}\right)+E_{\text {ex }}\left(L, X_{0}\right)
$$

with respect to $X_{0}$ and $L$.

For $x_{\mathrm{Mn}}=0$, and without Colomb interaction, the wavefunction in Eq. (A1) with $X_{0}=0$ is the exact singlet groundstate. The wavefunction can be generalized to the case of elliptical-confinement $\left(\omega_{x}<\omega_{y}\right)$ by replacing $L$ with the lengths $L_{x, y}=\sqrt{2} l_{x, y}$, the latter are defined in the main text. The energy of the singlet is $\hbar \omega_{x}+\hbar \omega_{y}$.

The variational wavefunction for the triplet in a circular QD is the same as in Eq. (4) in Ref. [A] 3$]$. The energy for the lowest triplet $(\mathrm{T})$ in an elliptical $\mathrm{QD}$ with $x_{\mathrm{Mn}}=0$ is obtained by placing one of the holes in the lowest excited orbital: $p_{x}$. The energy is then $E_{\mathrm{T}}=2 \hbar \omega_{x}+\hbar \omega_{y}$. Thus, the singlet-triplet gap decreases as $\hbar \omega_{x}=\hbar \omega_{0}(1+d)^{-2}$ with increasing $d$. The smaller this gap, the lower $x_{\mathrm{Mn}}$ value is sufficient for the triplet to become the groundstate ( $E_{\text {ex }}$ for the triplet is always more negative than $E_{\text {ex }}$ for PS). This fact can be used to understand the dependence of the PS-T boundary on increasing $d$, Fig. (3 main text).

Spin and charge densities for the triplet are proportional. Hence, the double-peaked spin density in Fig. (3b 
main text) can be explained in the variational model as a direct consequence of the form of triplet wavefunction: One hole is in the $s$ orbital, the other in the $p_{x}$ orbital.

\section{A1.1. Effective repulsion for Pseudosinglet}

It is instructive to fix $L$ and consider the trends of particular contributions to $E_{\text {tot }}$ [Eq. [A80] as a function of $X_{0}$. Kinetic energy does not depend on $X_{0}$, $E_{\text {kin }}=2 \hbar^{2} /\left(m^{*} L^{2}\right)$. Both Coulomb repulsion and Mnexchange terms decrease with increasing $X_{0}$, to zero and $-\Delta_{0}$, respectively. The latter limiting value can be understood as the energy of two independent magnetic polarons with opposite spin projections (hole spin-up and Mn spins-down for $x>0$, while hole spin down and Mn spins up for $x<0)$. This can be seen from $\rho_{\mathrm{PS}}(\boldsymbol{r})$ in Eq. (A7), which has a minimum and a maximum separated, in this limit, by $2 X_{0}$, see also Fig. (3a main text). Confinement (potential) energy increases as $m^{*} \omega_{0}^{2} X_{0}^{2}$, preventing the two holes from the complete separation, so that $X_{0}<+\infty$, and $\left|E_{\text {ex }}\left(L, X_{0}<\infty\right)\right|<\Delta_{0}$. Thus, we can treat $-\partial E_{\text {ex }}\left(L, X_{0}\right) / \partial X_{0} \propto \pm \Delta_{0} \exp \left[-2 X_{0}^{2} / L^{2}\right] / L$ as an effective repulsive force between the two holes. This force enhances the Coulomb repulsion.

The above reasoning remains qualitatively correct at non-zero temperatures, as long as they are are lower than the phase transition temperature. For $T>0$, we calculate the variational exchange energy from a generalization of Eq. (A6)

$$
E_{\mathrm{ex}}=-\Delta_{0} \int \rho_{\mathrm{s}}(\boldsymbol{r}) B_{S}\left(|\beta| \frac{5}{2} \frac{\rho_{\mathrm{s}}(\boldsymbol{r})}{h_{z} k_{B} T}\right) d^{2} r
$$

resulting from Eqs. (2, 6 main text). Eq. (A6) above is obtained in the limit of $T \rightarrow 0$, when the Brillouin function $B_{s}\left[|\beta| 5 \rho_{\mathrm{s}}(\boldsymbol{r}) /\left(2 h_{z} k_{B} T\right)\right] \rightarrow \operatorname{sign}\left[\rho_{\mathrm{s}}(\boldsymbol{r})\right]$.

Before numerically exemplifying the above model of effective interaction, we notice that the numerical holespin density $\left\langle s_{k}\right\rangle$ would not be a convenient choice for studying the effective repulsion in PS, as it vanishes in the small $x_{\mathrm{Mn}}$ limit. However, the spatial separation of the two holes can also be seen in the charge density (in units of $|e|)$

$$
\varrho(\boldsymbol{r})=\varphi_{u}(\boldsymbol{r})^{2}+\varphi_{d}(\boldsymbol{r})^{2} .
$$

The distance of any of the two symmetrical maxima from the QD center, $r=0$, is given by

$$
R_{\text {peak }}=X_{0} \tanh \left[\frac{4 R_{\text {peak }} X_{0}}{L^{2}}\right]
$$

in the variational framework. To satisfy this condition for $R_{\text {peak }}>0$, the condition $X_{0}>L / 2$ must hold. We neglect Coulomb interaction for simplicity for the rest of this subsection. For $x_{\mathrm{Mn}} \rightarrow 0$ we find $L \rightarrow L_{1}$, [Eq. A55], and $X_{0} \rightarrow 0$, so there is a lower-limit $x_{\mathrm{Mn}}$, below which $\varrho$ has only one extremum; a maximum at $r=0$. Above

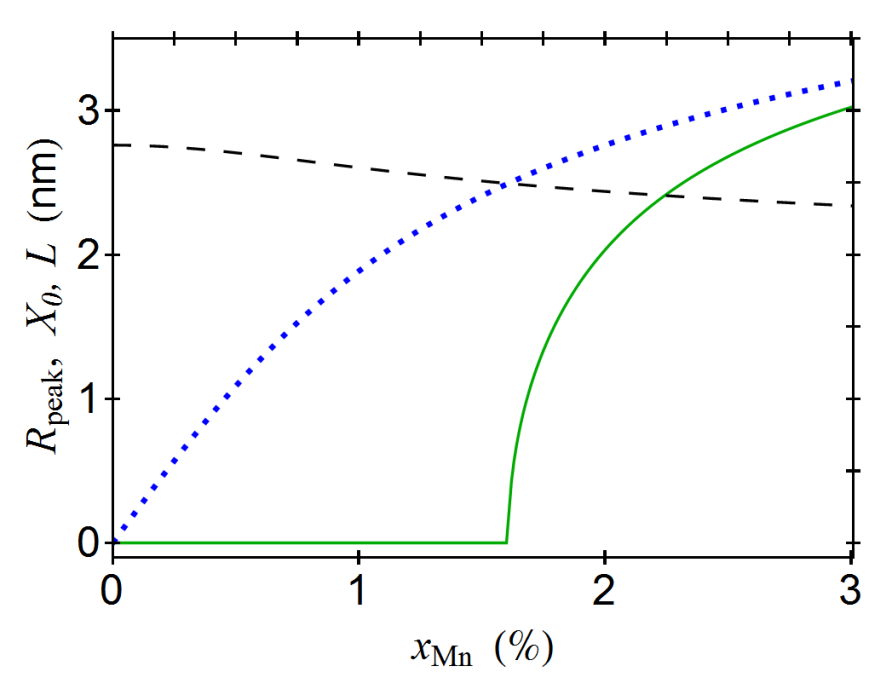

Figure A1. (color online) Variational results for positions of maxima of charge density $\varrho$ for PS at $T=0$. Solid green line: $R_{\text {peak }}$, dotted blue line: $X_{0}$, dashed black line: $L / 2$. We use the standard QD parameters defined in the main text, except for the effective Rydberg $R y^{*}=0$.

this $x_{\mathrm{Mn}}$, there are two maxima at $\boldsymbol{r}=\left( \pm R_{\text {peak }}, 0\right)$ and a minimum at $r=0$. The results of these considerations are shown in Fig. A 1

\section{A1.2. Effective attraction for Triplet}

In the case of the triplets discussed in the main text, the hole spin density $\rho_{\mathrm{T}}$ has a constant sign, so that $\int \rho_{\mathrm{T}} d^{2} r= \pm 1$. Thus, at zero temperature, the maximum energy gain occurs for unidirectional alignment of $\mathrm{Mn}$ spins, and no changes of the wavefunction are needed to attain the maximum, cf. Eq. (A6). This suggests that the $T=0$ limit is not helpful in the case of the triplets, since at any $T>0$, the local exchange energy, $|\beta| \rho_{\mathrm{s}}(\boldsymbol{r}) / h_{z}$, is smaller than $k_{B} T$ for Mn spins sufficiently remote from the center, so that some localization (change of wavefunction) is beneficial. As expected, the magnetization patterns for the triplets remain unidirectional at any temperature.

\section{A1.3. Effective interaction in the Exact Diagonalization method}

Figure $\mathrm{A} 2$ shows the numerical results obtained by the exact diagonalization (EXD) method described in the main text. The smallest Mn-content used is $x_{\mathrm{Mn}}=0.1 \%$. For this value, the number of Mn spins in the disk-shape volume of radius $L_{1}$ and height $h_{z}$ is $N=3$. Hence, for the very low contents $x_{\mathrm{Mn}} \leq 0.1 \%$, the continuum approximation would no longer be justifiable. Typical values of $x_{\mathrm{Mn}}$ in experiments are higher than $0.1 \%$. 


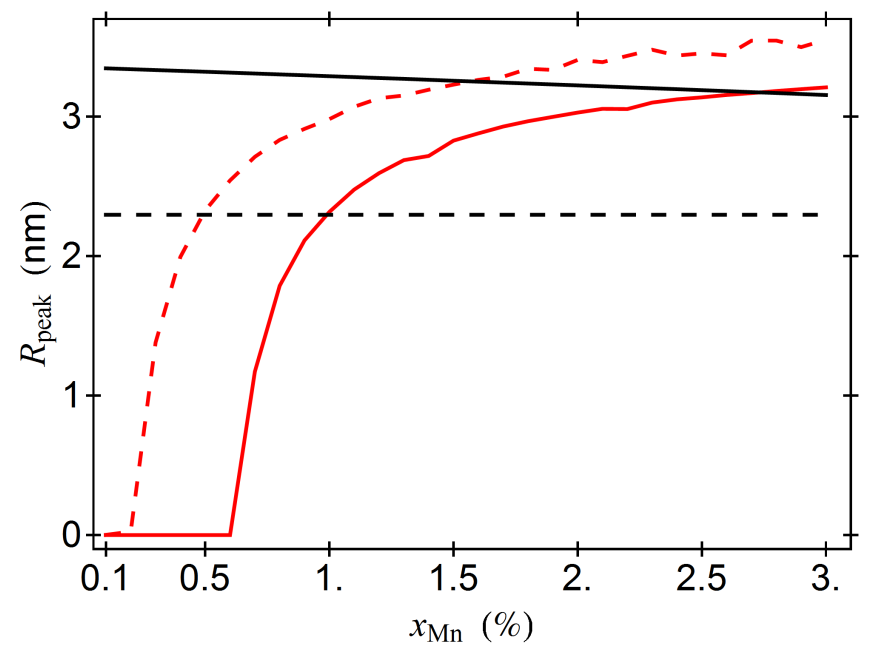

Figure A2. (color online) Distance, $R_{\text {peak }}$, from the center of a QD to the position of one of two symmetrical maxima in the charge density $\varrho$, calculated in EXD. $x_{\mathrm{Mn}}>0.1 \%$ as explained in the text here. Red: PS, black: triplet. Dashed: $T=0$, solid $T=2 \mathrm{~K}$. The oscillations at high $x_{\mathrm{Mn}}$ are artefacts due to numerical precision. We use the standard QD parameters defined in the main text, taking into account the Coulomb interaction.

Same as in the variational approach, we find that in EXD the distance $R_{\text {peak }}$ depends on $x_{\mathrm{Mn}}$, justifying the notion of effective interaction. Note the opposite behavior of PS vs. triplet at $T>0$, representing repulsion and attraction respectively, as discussed in the main text.

Comparing the EXD results for PS with the variational ones (Fig. A[1), we see that the off-center maxima start to appear at a much lower value of $x_{\mathrm{Mn}}$ (0.6 vs. 1.6\%). This is because the Coulomb interaction, present in EXD, favors separation of holes.

Finally, we note that a different quantum approach to interaction of magnetic bipolarons was used in Ref. [A 4$]$.

\section{A1.4. Core-halo pattern}

As explained in the main text, a different Mn-spin pattern forms for some Mn-doping profiles. This pattern can be studied variationally by replacing the $\varphi_{u}$ and $\varphi_{d}$ functions in Eq. (A1) by two Gaussians with $X_{0}=0$, but with different widths: $L_{u}$ for spin up and $L_{d}$ for spin down, see Eq. (3) in Ref. [A] 3$]$.

\section{A2. PHOTOLUMINESCENCE IN TYPE-II QDs}

In a $(\mathrm{Zn}, \mathrm{Mn}) \mathrm{Te} / \mathrm{ZnSe} \mathrm{QD}$ system, holes are confined in the QDs, while electrons are in the barrier (weakly bound to holes through Coulomb interaction) [A 5,6]. This is an example of the type-II band profile (Fig. A3), as opposed to the type-I profile, where both electrons and holes are confined. Thus, the exchange interaction of Mn with electrons is much weaker than with holes.

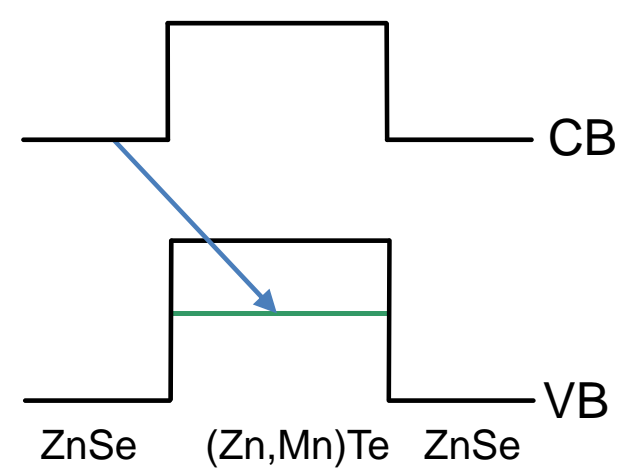

Figure A3. (color online) Band alignment for a type-II band profile, found in, e.g., ZnSe/ZnTe QDs. CB and VB stand for conduction and valence bands respectively.

Figure A4 shows the transitions calculated in the manuscript. We assume that owing to fast energy relaxation, luminescence from groundstates dominates over other initial states. Two types of transitions occur, in which the QD occupancies change as: $2 \rightarrow 1$ and $1 \rightarrow 0$. These two kinds of lines are separated by an energy determined mainly by hole repulsion (charging energy) $\sim 35$ $\mathrm{meV}$. In general, the final state of a $2 \rightarrow 1$ is not the initial state of the subsequent $1 \rightarrow 0$ transition, since relaxation of a Mn-spin pattern may occur between the two radiative events. The numerical calculations of the final states of the $2 \rightarrow 1$ transitions are not selfconsistent, but rather carried out for Mn-magnetization $m_{k}$ fixed in the pattern established when two holes are still present.

As argued in the main text, interband transitions from $p$-like states of electrons may arise due to breaking of the circular-symmetry by the Mn-pattern (the non-magnetic confinement potential remaining circularly symmetrical). The symmetry selection rules for the interband transitions are given by overlaps of electron and hole wavefunctions. Expanding the shifted Gaussian orbitals, Eqs. A2 A3 for small $X_{0} / L$, we obtain

$$
\begin{aligned}
& \varphi_{u}(\boldsymbol{r}) \simeq s(\boldsymbol{r})+\frac{X_{0}}{L} p_{x}(\boldsymbol{r}), \\
& \varphi_{d}(\boldsymbol{r}) \simeq s(\boldsymbol{r})-\frac{X_{0}}{L} p_{x}(\boldsymbol{r}),
\end{aligned}
$$

where $s=\sqrt{2 / \pi} \exp \left(-r^{2} / L^{2}\right) / L$, and $p_{x}=$ $\sqrt{8 / \pi} x \exp \left(-r^{2} / L^{2}\right) / L^{2}$. Thus, for $x_{\mathrm{Mn}}>0$, the hole wavefunction contains $p_{x}$ orbitals, which have the same symmetry as electron $p$-like orbitals. The hole wavefunction for the core-halo pattern does not have the $p$ orbitals in the equivalent expansion.

\section{ACKNOWLEDGMENTS}

We thank Jeongsu Lee and Karel Vyborny for help in calculations for this material. 


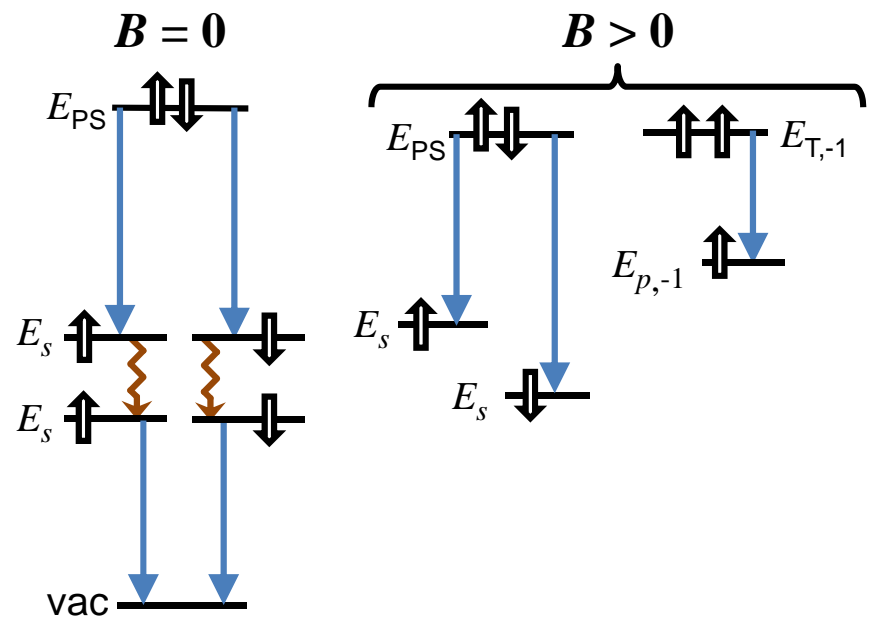

Figure A4. (color online) Jabłoński diagram [A 7] of the transitions discussed in the main text. Horizontal black lines: single and two-hole levels, as well as the zero-hole groundstate, "vacuum". Blue arrows: radiative transitions. Zig-zag arrows: non-radiative relaxation of $\mathrm{Mn}$ spins. Hollow arrows: spins of holes. An electron spin (not shown) must be antiparallel to hole spin to recombine [A 8] . The energies of the radiative transitions in the left/right panel are shown in Figs. $4 / 5$ of main text. $E_{\mathrm{PS}} / E_{\mathrm{T}}$ is the pseudo-singlet/triplet energy. $E_{s}$ or $E_{p}$ are the energies of a single hole in an $s$-like or $p$-like state respectively. The number -1 is the angular momentum of the triplet and $p$-like states.

A1. A. Szabo and N. Ostlund, Modern Quantum Chemistry (Dover Publications, 1989).

A2. See supplemental material at http://link.aps.org/supplemental/10.1103/PhysRevLett.

A3. R. Oszwałdowski, I. Zutić, and A. G. Petukhov, Phys. Rev. Lett. 106, 177201 (2011).

A4. H. Bednarski and J. Spałek, J. Phys.: Condens. Matter 24, 235801 (2012).

A5. I. R. Sellers, R. Oszwałdowski, V. R. Whiteside, M. Eginligil, A. Petrou, I. Žutić, W.-C. Chou, W. C. Fan, A. G.
Petukhov,

A6. I. L. Kuskovsky, W. MacDonald, A. O. Govorov, L. Mourokh, X. Wei, M. C. Tamargo, M. Tadic, and F. M. 6.177201eters, Phys. Rev. B 76, 035342 (2007).

A7. J. W. Lichtman and J.-A. Conchello, Nature Methods 2, 910 (2005).

A8. I. Žutić, J. Fabian, and S. Das Sarma, Rev. Mod. Phys. 76,323 (2004). 